\title{
On an identity of Ramanujan
}

\author{
Yoichi Motohashi
}

To the memory of S. Srinivasan

\begin{abstract}
Proofs published so far in articles and books, of the Ramanujan identity presented in this note, which depend on Euler products, are essentially the same as Ramanujan's original proof. In contrast, the proof given here is short and independent of the use of Euler products.
\end{abstract}

Keywords. Ramanujan's identity, Möbius inversion, Selberg's $\Lambda^{2}$-sieve.

2010 Mathematics Subject Classification. 11M06

This article is essentially a reproduction of the page 4 of [Mo09], for the sake of a wider audience. The identity of Ramanujan that we discuss here is

$$
\sum_{n=1}^{\infty} \frac{\sigma_{\alpha}(n) \sigma_{\beta}(n)}{n^{s}}=\frac{\zeta(s) \zeta(s-\alpha) \zeta(s-\beta) \zeta(s-\alpha-\beta)}{\zeta(2 s-\alpha-\beta)},
$$

where $\Re(s)>1+\max \{0, \Re \alpha, \Re \beta, \Re(\alpha+\beta)\}$; notations are as usual.

This famous formula is stated in [Ra1916] by Ramanujan; and his proof is in [Ra62, p. 135]. My aim is to give a new proof. It is short and, unlike Ramanujan's proof, avoids having to consider Euler products.

Proof. Note first that

$$
\sigma_{\alpha}(n) \sigma_{\beta}(n)=\sum_{u|n, v| n} u^{\alpha} v^{\beta}=\sum_{[u, v] \mid n} u^{\alpha} v^{\beta}
$$

where $[u, v]$ is the least common multiple of $u, v$. Thus the left side of (0.1) equals

$$
\zeta(s) \sum_{u, v} \frac{u^{\alpha} v^{\beta}}{[u, v]^{s}}=\zeta(s) \sum_{u, v} \frac{\langle u, v\rangle^{s}}{u^{s-\alpha} v^{s-\beta}},
$$

where $\langle u, v\rangle$ is the greatest common divisor of $u, v$. The Möbius inversion gives $\sum_{f \mid g} \eta_{s}(f)=g^{s}$ with $\eta_{s}(m)=\sum_{d \mid m} \mu(d)(m / d)^{s}$. Hence (0.3) equals

$$
\zeta(s) \sum_{u, v} \frac{1}{u^{s-\alpha} v^{s-\beta}} \sum_{d|u, d| v} \eta_{s}(d)=\zeta(s) \zeta(s-\alpha) \zeta(s-\beta) \sum_{d} \frac{\eta_{s}(d)}{d^{2 s-\alpha-\beta}} .
$$

The last sum is obviously equal to $\zeta(s-\alpha-\beta) / \zeta(2 s-\alpha-\beta)$. This completes the proof.

Remarks: Following kind recommendations of the reviewer I shall add some points closely related to the present subject.

(I) Methods: Treatments of (0.1) published so far in articles and books, save for the present one, are essentially the same as Ramanujan's original proof; thus they depend on Euler products. In contrast, the above proof is independent of Euler products; note that the application of the Möbius inversion immediately after (0.3) plays partly an alternative rôle. Any extension of the present method to general situations such as those dealt with in the recent article [SaRam16] which depends on Euler products appears to be an interesting arithmetical issue, especially in the automorphic context.

We thank episciences.org for providing open access hosting of the electronic journal Hardy-Ramanujan Journal 
(II) Applications: Ramanujan's identity extends readily to Dirichlet $L$-functions, which gives rise to a strikingly simple proof of Dirichlet's prime number theorem, a well known historical fact. This approach is pursued in [BaRa76] so that zero-free regions for Dirichlet $L$-functions are obtained without employing the Hadamard theory of entire functions. In [Mo83, Part II] their argument is combined with Selberg's $\Lambda^{2}$-sieve, which has yielded, among other things, an elementary proof of Vinogradov's zero-free region for the zeta-function and a drastically simplified (in fact, a new) proof of Linnik's famous zero-density theorem and Deuring-Heilbronn phenomenon for Dirichlet $L$ functions. Also, extensions of $(0.1)$ that involve $\left(\sigma_{\alpha}(n) \sigma_{\beta}(n)\right)^{2}$ and alike have important applications; for instance, in [4] a hypothetical improvement of Vinogradov's zero-free region is obtained; the hypothesis is of intriguing nature. As indicated above, in [SaRam16] an extension of Ramanujan's idea to automorphic $L$-functions is discussed in a general setting. Relevant to this, it should be remarked that the case of symmetric power $L$-functions is treated in [Mo15], and combined again with the $\Lambda^{2}$-sieve new assertions are established on the distribution of zeros of those $L$-functions and consequently of Hecke eigenvalues over primes in short intervals.

Acknowledgements: I am highly grateful to the reviewer for sending me the copies of [BaRa76] and [SaRam16]. I thank Nigel Watt for his kind comments on the draft of the present article.

\section{References}

[BaRa76] R. Balasubramanian and K. Ramachandra, A place of an identity of Ramanujan in prime number theory, Proc. Indian Acad. Sci., 83A (1976), 156-165.

[SaRam16] Sanoli Gun and M. Ram Murty, Generalization of an identity of Ramanujan, J. Ramanujan Math. Soc., 31 (2016), $125-135$.

[Mo83] Y. Motohashi, Lectures on sieve methods and prime number theory, Tata Inst. Fund. Res. Lect. Math. Phy., 72, TIFR, Bombay \& Springer Verlag, Berlin, 1983.

[Mo01] Y. Motohashi, An observation on the zero-free region of the Riemann zeta-function, Periodica Math. Hungarica, 42 (2001), 117-122.

[Mo09] Y. Motohashi, Analytic number theory I: The theory of the distribution of prime numbers Asakura Publishing, Tokyo, 2009. (Japanese: a far enlarged English version is to be published).

[Mo15] Y. Motohashi, On sums of Hecke-Maass eigenvalues squared over primes in short intervals, J. London Math. Soc., (2) 91 (2015), 367-382.

[Ra1916] S. Ramanujan, Some formulae in the analytic theory of numbers, Mess. Math., 45 (1916), 81-84.

[Ra62] S. Ramanujan, Collected papers Chelsea, New York, 1962.

\section{Yoichi Motohashi}

Honkomagome 5-67-1-901

Tokyo 1130021, JAPAN.

e-mail: ymoto@math.cst.nihon-u.ac.jp 